\title{
Current status of the verification and processing system GALILÉE-1 for Evaluated Data
}

\author{
Mireille Coste-Delclaux ${ }^{1}$, Cédric Jouanne ${ }^{1, *}$, Frédéric Moreau ${ }^{1}$, Claude Mounier ${ }^{1}$, Thierry \\ Visonneau ${ }^{1}$, and Fadhel Malouch ${ }^{1}$ \\ ${ }^{1}$ DEN, Service d'Études des Réacteurs et de Mathématiques Appliquées (SERMA), CEA, Université \\ Paris-Saclay, F-91191, Gif-sur-Yvette, France
}

\begin{abstract}
This paper describes the current status of GALILÉE-1 that is the new verification and processing system for evaluated data, developed at CEA. It consists of various components respectively dedicated to $\mathrm{read} /$ write the evaluated data whatever the format is, to diagnose inconsistencies in the evaluated data and to provide continuous-energy and multigroup data as well as probability tables for transport and depletion codes. All these components are written in $\mathrm{C}++$ language and share the same objects. Cross-comparisons with other processing systems (NJOY, CALENDF or PREPRO) are systematically carried out at each step in order to fully master possible discrepancies. Some results of such comparisons are provided.
\end{abstract}

\section{Introduction}

GALILÉE-1 system, written in C++ language is a new verification and processing system for evaluated data. It is part of a CEA global development program dedicated to fine modelling of nuclear systems. At the present time, three main components are under development:

- GALION (GALilée Input Output for Nuclear data): dedicated to read evaluated data and write produced data.

- GALVANE (GALilée Verification of the Accuracy of Nuclear Evaluations): dedicated to verify nuclear evaluations that are GALILÉE-1 input data.

- GTREND (Galilée TReatment of Evaluated Nuclear Data): dedicated to provide continuous-energy (CE) and multigroup (MG) data as well as probability tables (PT).

Additional components, such as interface modules creating consistent libraries for application codes or a convivial and automatic chain for creating these libraries, will be developed later.

GALILÉE-1 system originality lays in its two complementary running ways: an integrated one and an open one for providing application codes with processing tools.

* Corresponding author: cedric.jouanne@,cea.fr 


\section{GALILÉE-1 system description}

GALILÉE-1 system is built upon GBASE component that defines and implements a set of common objects, shared by all other GALILÉE-1 components. GBASE objects are completely independent from the input and output data formats.

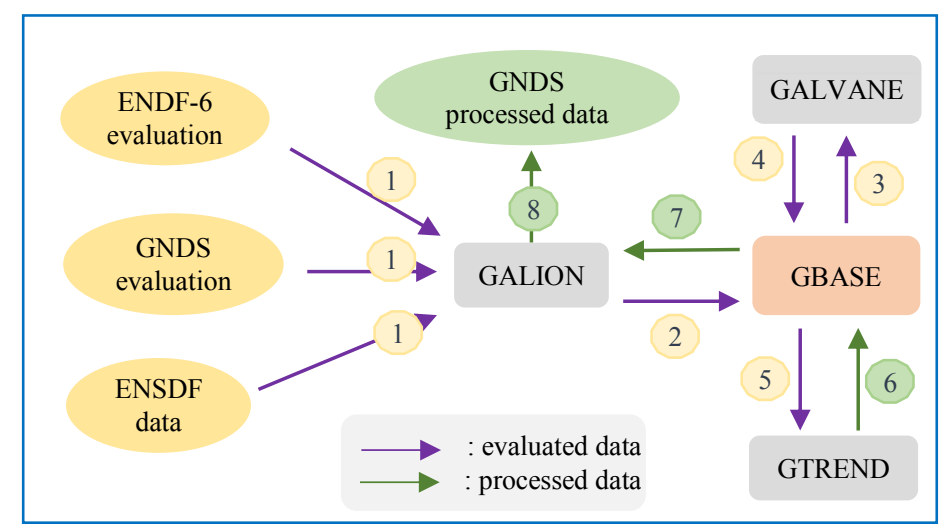

Fig. 1. GALILÉE-1 processing modules.

As shown in Figure 1, GBASE objects are initialized thanks to GALION that reads the evaluation or the structure data. These objects are checked and possibly corrected by GALVANE and then processed data are created by GTREND. One has to note that GALVANE and GTREND only work on GBASE objects, which allows the same verification and processing stages, whatever the evaluation format is. The objects storing processed data are kept in GBASE and can be written on binary or ASCII files by GALION.

\section{GBASE Objects}

The GBASE object hierarchy is very close to the GNDS object hierarchy. For each nucleus or element, we create a database allowing us to store, in the same object, structure data and interaction data for a given projectile. "GBASE structure data" contain all the information needed to verify and optionally correct the evaluated data: masses, level scheme, spins, energy, half-life, decay modes, etc. "GBASE interaction data" contain:

- the list of products that can be created by the interaction,

- all the information given in an evaluation (JEFF-3.3, ENDF/B-VIII, JENDL$4.0, \ldots)$ but organized in such a way that processing is easier,

- data processed using GALILÉE-1 (CE data, Probability Tables, MG data, ...).

Several GBASE structure data or several GBASE interaction data may exist in the same database.

\section{GALION Module}

GALION can read evaluated data in ENDF-6 or GNDS (under progress) format as well as structure data in ENSDF or NUBASE format. It supplies tools for creating the GBASE objects corresponding to structure data or evaluated data. It also provides tools for writing continuous energy data in PENDF format. 


\section{GALVANE Module}

One of the goals of GALILÉE-1 system is to test the consistency and the validity of nuclear data evaluations. We plan to perform a complete assessment of evaluated files before any treatment. Currently, GALVANE can diagnose inconsistencies in general information, resonance parameters, $\mathrm{Q}$ reaction values, thresholds, excited level schemes, kinematic data of emitted particles and thermal scattering laws. Some checks can be performed by comparing data with the ones contained in structure databases, e.g. NUBASE or ENSDF. This is the case for:

- masses of nuclides, given in terms of neutron mass,

- energies of excited states reached in the inelastic scattering,

- gamma decay schemes of the excited states.

Some additional tests are designed to check the consistency between the data given in an evaluation:

- consistency between thresholds considered for various data of the same reaction,

- energy balance for reaction products,

- spin/parity of resonance parameters,

- normalization of distributions.

\section{GTREND Module}

GTREND code aims at replacing NJOY [1] and CALENDF [2] codes in CEA application library production. It consists of three main parts, GTREND_CE corresponding to NJOY/RECONR, /BROADR, /UNRESR, /THERMR and /HEATR, GTREND_PT corresponding to CALENDF and GTREND_MG corresponding to NJOY/GROUPR.

Today, GTREND can reconstruct continuous energy cross-sections in the resolved resonance range, averaged cross-sections in the unresolved resonance range, generate a linearization grid, broaden linearized cross-sections, and calculate moment based probability tables.

\subsection{Reconstruction in the resolved resonance range}

\section{Formalism}

Following references [3], [4] and [5], in scattering theory, a channel $c$ is characterized by the pair $\alpha$ of two particles making up the channel, the orbital angular momentum of the pair $\ell$, the channel spin $s$ (including associated parity) that is the sum of the spins of the two particles of the pair and the total angular momentum $J$ (including associated parity). A spin group of channels is defined as a set of channels with the same total angular momentum $J$. The angle-integrated cross-section from entrance channel $c$ to exit channel $c^{\prime}$, with total angular momemtum $J$, is given, in terms of the scattering matrix $U_{c c}$, by:

$$
\sigma_{c, c^{\prime}}=\frac{\pi}{k_{\alpha}^{2}} g_{J \alpha}\left|e^{2 i w_{c}} \delta_{c C^{\prime}}-U_{c c^{\prime}}\right| \delta_{J J}
$$

where $k_{\alpha}$ is the wave number, $g_{J \alpha}$ the spin statistical factor and $w_{c}$ is the difference between the Coulomb phase shift for a given $\ell$ and for $\ell=0$ (this difference is equal to zero for non-Coulomb channels). 
The scattering matrix $U$, that describes the transition between entrance and exit channels, can be written:

$$
U=\Omega\left[1+2 i P^{\frac{1}{2}}(1-R L)^{-1} R P^{\frac{1}{2}}\right] \Omega
$$

- $\Omega$ is the diagonal matrix which diagonal term is given by $\left.\Omega_{c, c}=e^{-i\left(w_{c}-\varphi_{c}\right.}\right), \varphi_{c}$ being the potential-scattering phase shift,

- $L$ is the diagonal matrix which diagonal term is given by $L_{c, c}=S_{c}+i P_{c}-B_{c}, S_{c}, P_{c}, B_{c}$ being respectively the shift factor, the penetrability and a boundary condition (real functions),

- $P$ is the diagonal matrix which diagonal term is equal to $P_{c}$,

- $R$ is the channel matrix which terms are defined by:

$$
\mathrm{R}_{c, c \prime}=\sum_{\lambda} \frac{\gamma_{\lambda, c} \gamma_{\lambda, c l}}{E_{\lambda}-E} \delta_{J J}
$$

$\gamma_{\lambda, c}$ standing for the channel amplitude, $E_{\lambda}$ for the energy of the level, $E$ for the neutron kinetic energy and $J$ and $J^{\prime}$ for respectively the total angular momentum (with associated parity) of the channel $c$ and $c^{\prime}$.

The scattering matrix $U$ can be written in terms of $X$ matrix as:

$$
\begin{aligned}
& U=\Omega[1+2 i X] \Omega \\
& X=P^{\frac{1}{2}}(1-R L)^{-1} R P^{\frac{1}{2}}
\end{aligned}
$$

In GTREND, the angle integrated cross-section for the interaction leading from particle pair $\alpha$, for which one particle is a neutron, to particle pair $\alpha^{\prime}$, is deduced from $\mathrm{X}$ matrix in the following way:

$\sigma_{\alpha \alpha^{\prime}}=\frac{4 \pi}{k_{\alpha}^{2}} \sum_{J} g_{J \alpha} \sum_{c}\left[\left(\sin ^{2}\left(\varphi_{c}\right)\left(1-2 \operatorname{Im}\left(X_{c c}\right)\right)-\sin \left(2 \varphi_{c}\right) \operatorname{Re}\left(X_{c c}\right)\right) \delta_{c \prime^{\prime}}+\sum_{c \prime}\left|X_{c c \prime^{\prime}}\right|^{2}\right]$

The summations are over channels $c$ and $c^{\prime}$ belonging to the spin group $J$ and such that the particle pair is $\alpha$ for $c$ and $\alpha^{\prime}$ for $c^{\prime}$.

The nuclear formalisms currently supported in GTREND are Single and Multi-level Breit-Wigner, Reich-Moore, and R-Matrix-Limited formalisms. The classical approximations are implemented.

\section{Results}

In order to validate GTREND reconstruction at 0 Kelvin in the resolved resonance range, cross-comparisons with NJOY2016 were carried out for all JEFF-3.2 nuclei $(\sim 470)$. About 25 nuclei show relative reconstruction discrepancies larger than $10^{-5}$, between NJOY2016 and GTREND, for MT1, MT2, MT102 and MT18 when it exists. All observed discrepancies can be explained. Figure 2 shows a comparison of the cross-section reconstructions with NJOY2016 and GTREND. 

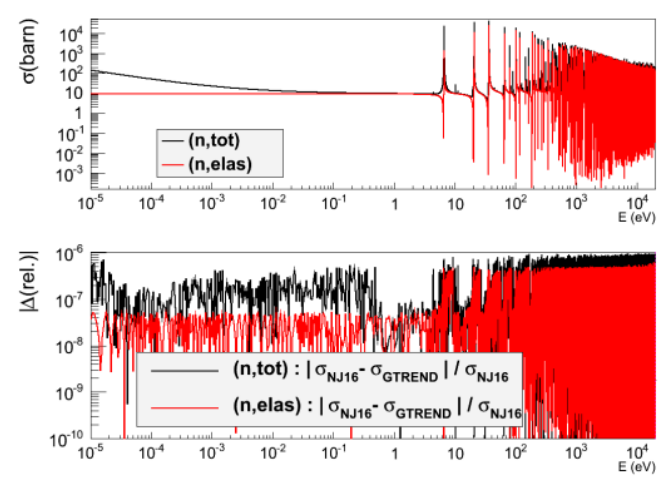

Fig. 2. Comparison between NJOY2016 and GTREND on U238 reconstruction in RRR

\subsection{Linear piecewise reconstruction}

A tool building a piecewise linear approximation of the cross-sections derived from a nuclear formalism (accurate but time consuming) is very convenient and efficient for the processing itself and for Monte Carlo transport codes. In the frame of GALILÉE-1, we developed a generic linearization tool. Various concrete criteria are implemented such as Punctual, Integral or Mixed ones. When an integral criterion is used a weighting function $\mathrm{W}$ is mandatory.

The function $\mathrm{F}$ to be represented in a piecewise linear form has to be a "functor" with a specific signature. This allows us to use the same algorithm for various types of functions: resonance formalism, thermal scattering model, Legendre expansion of an angular distribution or tabulated function with various interpolation schemes. The user must give, as input data, well-chosen points in the linearization range.

The basis of the GALILÉE-1 algorithm is very similar to the one implemented in NJOY: halving interval and checking with some kind of criteria. The first two basic criteria are the maximum number of subdivisions of an initial interval and the minimum width of an interval expressed in term of a multiple of the machine epsilon. All the specialized criteria use these two parameters.

A convergence diagnostic is available to check if the "convergence" is reached using these criteria. The diagnostic can be used to trigger a restart of the linearization process with the same or with modified parameters of the criterion. This restart mode is useful if the user cannot set properly the initial points of the linearization.

\subsection{Doppler broadening}

\section{Description}

The Doppler broadening and the thermal modules are designed consistently in GTREND whatever the thermal motion is (free gas or chemical binding model). The Doppler broadening and the calculation of thermal scattering cross-sections can be done starting from a nuclear cross-section given by the true formalism or from a linearized one. At present time, only the "SIGMA1" method designed in PREPRO [5] system that provides an exact Doppler broadening for a piecewise linear representation of a cross-section, is implemented in GTREND. 


\section{Results}

For validating GTREND, we performed, on U238 JEFF-3.2, a SIGMA1 Doppler broadening at $300 \mathrm{~K}$ on the NJOY2016/BROADR energy grid and we compared the crosssections to the NJOY ones. The relative discrepancies are given in Figure 3. The general trend is satisfactory but at high energies, NJOY2016 curve may display unphysical behaviors leading to higher discrepancies.

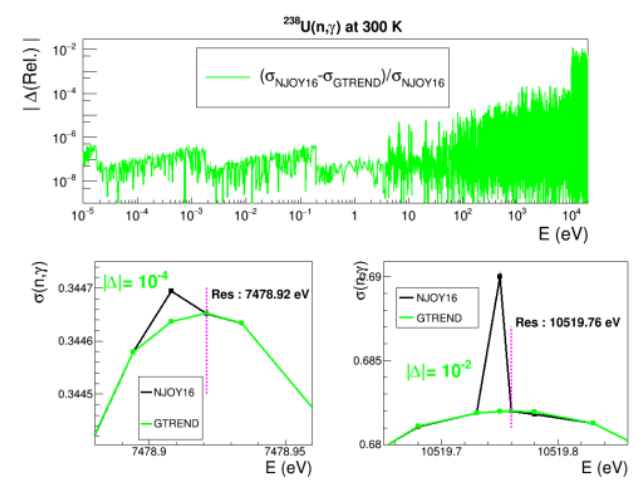

Fig. 3. Relative discrepancies on radiative capture cross-sections of U238 at $300 \mathrm{~K}$

\subsection{Probability Table calculation in the Unresolved Resonance Range (URR) on an evaluator-defined energy grid}

In this section, we present the first results on probability table calculations in URR. The use of the average data available in the evaluation files requires the sampling of quantities using several distribution laws. Spacing between the resonances is distributed according to the Wigner's law. Partial widths are represented by $\chi$-2's laws with various degrees of freedom.
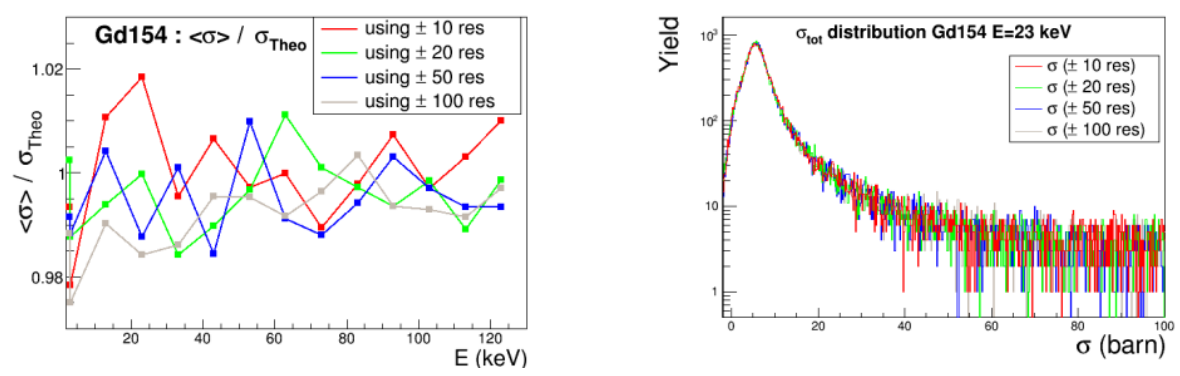

Fig. 4. 4a (left): Ratio between average total cross-section from 30,000 sets of resonances and theoretical cross-section using different number of resonances at $300 \mathrm{~K}$. $4 \mathrm{~b}$ (right): Histogram of 30,000 total cross-sections using different numbers of resonances.

We have undertaken various tests to validate the calculation of these probability tables. In a first time, we sampled 30,000 sets of random resonances to define the average crosssections on an energy grid in the unresolved domain. The nucleus selected is Gd154 from JEFF-3.2 library that contains URR from $2.76 \mathrm{keV}$ to $123 \mathrm{keV}$. We were concerned with the influence of the number of resonances taken into account around the energetic position for the calculations. We sequentially considered 20, 40, 100, and 200 resonances around this computational energy. Figure 4a shows the ratio between the average values calculated for these 30,000 sets of resonances and the theoretical average value as a function of the 
number of resonances. Figure $4 \mathrm{~b}$ shows the histograms of the 30,000 total cross-section values calculated at $23 \mathrm{keV}$ for this nucleus as a function of the number of resonances taken into account. These histograms are the input for the different probability table calculation.

Figure 5a presents these calculated probability tables for an incident energy of $23 \mathrm{keV}$ and compares them to the one processed by NJOY's PURR module. We observe a very good agreement for the various probability tables produced with GTREND independently of the number of resonances taken into account. The comparison with the NJOY2016/PURR probability table shows a slightly different distribution, in particular an extension to higher cross-sections in the case of calculations provided by GTREND.
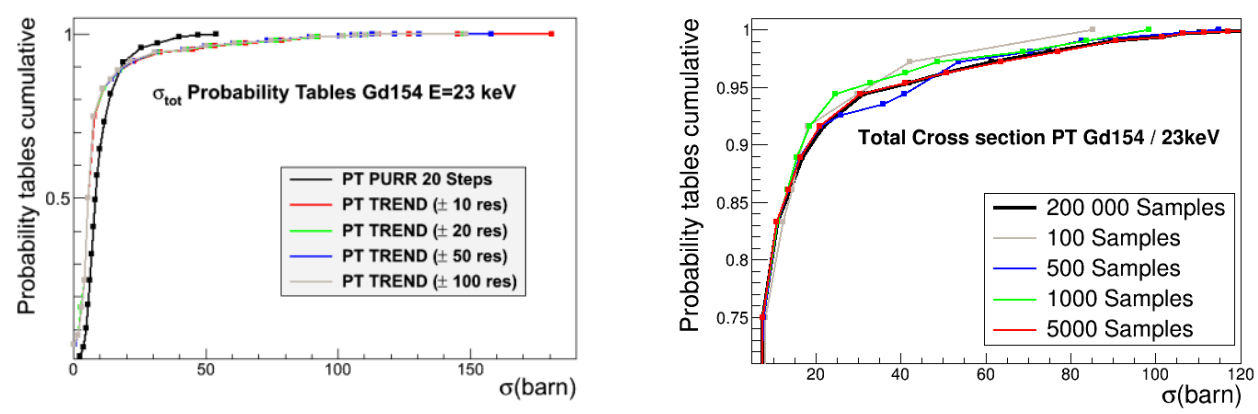

Fig. 5. 5a (left): Comparison of probability table cumulative distributions from PURR and GTREND using various numbers of resonances. $5 \mathrm{~b}$ (right): Comparison of probability table cumulative distributions calculated with GTREND as a function of the number of resonance sets.

An important question is to define the number of resonance sets to be taken into account in order to establish a converged probability table. To do this, we sampled several series of 100 resonances around $23 \mathrm{keV}$. Figure $5 \mathrm{~b}$ shows the evolution of probability tables as a function of the number of resonance sets. The reference is a calculation performed using 200,000 sets of resonances. We observe that from 5,000 sets of resonances the agreement becomes accurate with the reference.

These various tests must be continued and improved in order to obtain a systematic treatment for the calculation of probability tables for all nuclei containing an unresolved resonance domain.

\section{Conclusion}

GALILÉE-1 system will have soon the capability of producing Monte Carlo transport libraries from validated evaluations, which is a first important step for our renewed library creation chain. It allows R\&D activity on new processing methods and is also a powerful tool for nuclear data analysis.

\section{References}

1. R.E. MacFarlane et al., LANL, USA, No. LA-UR-17-20093, (2017)

2. J.C. Sublet, P. Ribon, and M. Coste-Delclaux, CEA-R-6277, (2011)

3. A. Lane and R. Thomas, Rev. Mod. Phys., 30, 257, (1958)

4. A. Trkov, M. Herman and D. A. Brown, Report BNL-90365-2009 Rev.2, (2012)

5. N. Larson, ORNL/TM-9179/R8, (2008)

6. D. E. Cullen, ORNL, No. PREPRO2012, 004357WKSTN00, USA (2015). 